\title{
Identification of quantitative trait loci for resistance to two species of root-lesion nematode (Pratylenchus thornei and P. neglectus) in wheat
}

\author{
R. S. Zwart ${ }^{\mathrm{A}, \mathrm{B}}$, J. P. Thompson ${ }^{\mathrm{A}, \mathrm{C}}$, and I. D. Godwin ${ }^{\mathrm{B}}$

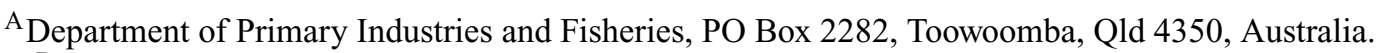 \\ ${ }^{B}$ School of Land and Food Sciences, University of Queensland, Brisbane, Qld 4072, Australia. \\ ${ }^{\mathrm{C}}$ Corresponding author. Email: john.thompson@dpi.qld.gov.au
}

\begin{abstract}
Pratylenchus thornei and P. neglectus are two species of root-lesion nematode that cause substantial yield losses in wheat. No commercially available wheat variety has resistance to both species. A doubled-haploid population developed from a cross between the synthetic hexaploid wheat line CPI133872 and the bread wheat Janz was used to locate and tag quantitative trait loci (QTLs) associated with resistance to both P. thornei and P. neglectus. Wheat plants were inoculated with both species of nematode in independent replicated glasshouse trials repeated over 2 years. Known locations of wheat microsatellite markers were used to construct a framework map. After an initial single-marker analysis to detect marker-trait linkages, chromosome regions associated with putative QTLs were targetted with microsatellite markers to increase map density in the chromosome regions of interest. In total, 148 wheat microsatellite markers and 21 amplified fragment length polymorphism markers were mapped. The codominant microsatellite marker Xbarc183 on the distal end of chromosome 6DS was allelic for resistance to both P. thornei and P. neglectus. The QTL were designated QRInt.lrc-6D.1 and QRInn.lrc-6D.1, for the 2 traits, respectively. The allele inherited from CPI133872 explained $22.0-24.2 \%$ of the phenotypic variation for $P$. thornei resistance, and the allele inherited from Janz accounted for 11.3-14.0\% of the phenotypic variation for P. neglectus resistance. Composite interval mapping identified markers that flank a second major QTL on chromosome 6DL (QRlnt.lrc-6D.2) that explained 8.3-13.4\% of the phenotypic variation for $P$. thornei resistance. An additional major QTL associated with $P$. neglectus resistance was detected on chromosome 4DS (QRlnn.lrc-4D.1) and explained a further $10.3-15.4 \%$ of the phenotypic variation. The identification and tagging of nematode resistance genes with molecular markers will allow appropriate allele combinations to be selected, which will aid the successful breeding of wheat with dual nematode resistance.
\end{abstract}

Additional keywords: synthetic hexaploid wheat, doubled haploids, composite interval mapping.

\begin{abstract}
Introduction
Two species of root-lesion nematode, Pratylenchus thornei Sher and Allen and P. neglectus (Rensch) Filipjev and Schuurmans Stekhoven, cause substantial yield losses in cereal crops throughout the world. High populations of P. thornei cause up to $85 \%$ yield loss in wheat (Triticum aestivum L.) varieties (Thompson et al. 1993). Similarly, $P$. neglectus causes up to $27 \%$ yield loss in wheat varieties (Taylor et al. 1999). In Australia, annual lost wheat production caused by $P$. thornei and $P$. neglectus is estimated at $\$ 36$ million and $\$ 12$ million, respectively (Brennan and Murray 1998).

Glasshouse screening of wheat lines inoculated with pure cultures of nematodes has suggested that the genetic mechanisms that confer resistance to $P$. thornei do not confer resistance to P. neglectus (Farsi et al. 1995; Taylor et al.
\end{abstract}

1999). However, mixed populations of both species can occur in the same field and therefore breeding wheat varieties with resistance to both species of nematode is a desirable goal. Unfortunately, selecting resistant wheat lines in a conventional breeding program relies on the time-consuming and laborious procedure of inoculating plants in the glasshouse and then extracting and counting the nematodes that have multiplied in the plant roots. Implementing marker-assisted selection for root-lesion nematode resistance could greatly accelerate breeding for multiple disease resistance.

Single resistance genes and quantitative trait loci (QTLs) associated with disease resistance traits have been characterised in wheat by using either full genetic linkage maps or the rapid and cost-effective approach of bulked segregant analysis (BSA) (Michelmore et al. 1991). In recent 
studies, microsatellite (simple sequence repeat, SSR) markers have emerged as the marker system of choice because of their abundance throughout the wheat genome, high level of polymorphism, and genome specificity (Langridge et al. 2001). Amplified fragment length polymorphism (AFLP) markers are another robust and reliable technique widely used as they have the potential to generate a large number of polymorphic loci in a single assay (Langridge et al. 2001).

The genetic basis for resistance to $P$. thornei in synthetic hexaploid wheat lines has been shown to be polygenic and additive (Zwart et al. 2004a). However, to date, only limited sources of resistance to root-lesion nematodes in wheat have been investigated at the molecular level. QTLs for resistance to $P$. thornei were identified in the International Triticeae Mapping Initiative (ITMI) population on chromosomes 6D and 2B (Thompson et al. 1999). A single gene for P. neglectus resistance, Rlnn1, has been mapped to chromosome 7AL in a Tammin $\times$ Excalibur cross (Williams et al. 2002). Markers linked to Rlnn1 in this population were the microsatellite marker Xgwm344, the flour colour PCR marker $X s c h f c 3$, co-segregating restriction fragment length polymorphism (RFLP) markers Xcdo347, Xpsr680A, and Xpsr121, and AFLP marker XAGC/CCT179 (Williams et al. 2002). Identification of novel sources of resistance and further understanding of the genetics of different sources of resistance will enable combinations of resistance genes to be pyramided, producing wheat varieties with durable multiple disease resistance.

It has been suggested that 8000 years ago, when hexaploid wheat (AABBDD, $2 n=6 \mathrm{x}=42$ ) first appeared, only a limited number of genotypes from both the diploid Aegilops tauschii Coss. (DD, $2 n=2 \mathrm{x}=14)$ and tetraploid Triticum turgidum L. subsp. durum (Desf.) Husn (AABB, $2 n=4 \mathrm{x}=28$ ) wild progenitor species were involved in the natural intercrossing (Breiman and Graur 1995). Thus, hexaploid bread wheat lacks the rich diversity in resistance genes that exists in its tetraploid and diploid ancestors. Diverse accessions of Ae. tauschii with resistance to $P$. thornei have been identified (Thompson and Haak 1997), and these sources of resistance can be exploited by hybridising tetraploid and diploid relatives of wheat to make synthetic hexaploid wheat. Recently, very effective sources of resistance to both $P$. thornei and P. neglectus were identified in synthetic hexaploid wheat lines (Zwart et al. 2004b).

The objective of this research was to identify and map QTLs associated with resistance to both $P$. thornei and $P$. neglectus in a population derived from a synthetic hexaploid wheat line. Knowing the locations of genes that confer resistance to $P$. thornei and $P$. neglectus will give insight into the most effective breeding strategy to obtain durable resistance to both species of root-lesion nematode.

\section{Materials and methods}

\section{Plant material and DNA extraction}

The mapping population consisted of $100 \mathrm{~F}_{1}$-derived doubled-haploid (DH) lines derived from the cross of CPI133872, a resistant synthetic hexaploid wheat, and Janz, a susceptible prime hard bread wheat. The DH lines were produced using wheat $\times$ maize hybridisation (Kammholz et al. 1996). Deoxyribonucleic acid was extracted from leaf tissue using a modified sarkosyl protocol (Rogowsky et al. 1991).

\section{Root-lesion nematode screening}

The lines were phenotyped for resistance to $P$. thornei and $P$. neglectus in separate glasshouse experiments duplicated in consecutive years. In 2001, 6 replicates of $100 \mathrm{DH}$ lines were evaluated for resistance to P. thornei and 3 replicates of $90 \mathrm{DH}$ lines were screened for resistance to $P$. neglectus. In 2002, 6 replicates of $100 \mathrm{DH}$ lines were screened for resistance to both $P$. thornei and $P$. neglectus. All experiments were grown in Latinised row-column designs. Nematode experiments were evaluated following the procedure of Thompson et al. (1999) with modifications as described by Zwart et al. (2004a).

\section{Microsatellite analysis}

In total, 289 microsatellite primer pairs were used to screen the parents for polymorphisms: 151 GWM (Röder et al. 1998), $68 \mathrm{WMC}$ (Prasad et al. 2000; Gupta et al. 2002), 26 PSP (Bryan et al. 1997; Stephenson et al. 1998), 23 BARC (Cregan 2001), 19 GDM (Pestsova et al. 2000), 1 CFD (Guyomarc'h et al. 2002), and 1 GPW primer pair (Berry 2002). BSA was used for the screening of 238 microsatellite primer pairs. An additional 51 microsatellite primer pairs, selected from published maps for targetted mapping of specific chromosome regions, were screened over the parents alone. PCR amplification was performed in a volume of $10 \mu \mathrm{L}$ in a MJ research thermocycler. Each reaction mixture contained $1 \times$ PCR buffer (Invitrogen), $1.5 \mathrm{~mm}$ $\mathrm{MgCl}_{2}$ (Invitrogen), $0.2 \mathrm{~mm}$ each deoxynucleotide (Progen), $250 \mathrm{~nm}$ each forwards and reverse microsatellite primer (Proligo), $1 \mathrm{U}$ Taq (Invitrogen), and $100 \mathrm{ng}$ genomic DNA. Standard PCR thermal cycling procedures were performed using an initial denaturation step of $5 \mathrm{~min}$ at $94^{\circ} \mathrm{C}$, followed by 35 cycles at $94^{\circ} \mathrm{C}$ for $1 \mathrm{~min} ; 30 \mathrm{~s}$ at 50,55 , or $60^{\circ} \mathrm{C}$, depending on the primer pair; $30 \mathrm{~s}$ at $72^{\circ} \mathrm{C}$; and a final elongation step of $72^{\circ} \mathrm{C}$ for $5 \mathrm{~min}$. PCR products were separated using either $3 \%$ high-resolution agarose gels (Progen) stained with ethidium bromide or $6 \%$ non-denaturing polyacrylamide gels visualised with silver staining (Bassam and Caetano-Annollés 1993).

\section{AFLP analysis}

AFLP analysis was performed according to the method of Vos et al. (1995) using PstI and MseI restriction enzymes (New England Biolabs) for the rare and frequent cutters, respectively, as described by Campbell et al. (2001). AFLP primer combinations were screened using BSA. The 5 most polymorphic Pst I/MseI AFLP primer combinations (P33M53, P33M54, P35M47, P37M54, and P45M53) were used to selectively amplify all DH lines in the population. AFLP products were run on $6 \%$ denaturing polyacrylamide gels and visualised by silver staining.

\section{BSA and framework map}

Equal quantities of DNA from 10 lines, at the extremes of phenotype variation for each of the nematode species, were pooled to form resistant and susceptible bulks (Michelmore et al. 1991). Microsatellite and AFLP primers were screened to identify markers that were polymorphic between the parents and bulks. Polymorphic markers were then used to genotype the $100 \mathrm{DH}$ lines and to test for an association with resistance to root-lesion nematodes. 
In addition, the BSA primer screen identified microsatellite markers polymorphic between the parents used to construct the framework map. Published chromosome assignments of these markers were used to select markers to be mapped across the entire population, with the aim of obtaining at least 1-2 microsatellite markers per chromosome arm for the 21 chromosomes of wheat. Chromosome regions with putative associations to resistance to $P$. thornei and P. neglectus were then targetted with additional microsatellite markers to increase map density in these areas of interest. The PCR marker $X s c h f c 3$ (Williams et al. 2002) was screened across the entire mapping population using conditions as described above for microsatellite analysis. Chi-square analyses were applied to test the goodness-of-fit of the observed segregation ratios to theoretical expectations of the Mendelian segregation ratio $(1: 1)$.

Linkage analyses were performed using the software packages MapMaker/exp (Lander et al. 1987) and MapManager (Manly and Elliott 1991), with minimum logarithm of the odds ratio (LOD) score of 3.0 and the Kosambi mapping function (Kosambi 1944). Known locations of the chromosome-specific microsatellite markers were used as a reference to incorporate markers unlinked at the criterion LOD of 3.0 into the framework map at the most likely interval.

\section{QTL analysis}

Spatially adjusted line means were used to detect QTLs using QTL Cartographerv2.0 (Wang et al. 2004). A preliminary scan of the genome using statistical associations based on single-markers was carried out to identify putative QTL regions. The positions and effects of QTLs were determined using simple interval mapping and composite interval mapping. The significant threshold likelihood ratio statistic (LRS) for detection of QTLs was calculated for each set of phenotypic data using 1000 permutations at $P<0.05$ (Churchill and Doerge 1994). The proportion of observed phenotypic variation explained due to a particular QTL was estimated by the coefficient of determination $\left(R^{2}\right)$. Composite interval mapping was performed using forwards-backward stepwise regression with a window size of $10 \mathrm{cM}$ and background control set at 5 markers.

\section{Results}

\section{Root-lesion nematode phenotyping}

The DH population showed continuous distribution for resistance to both $P$. thornei and $P$. neglectus (Fig. 1).
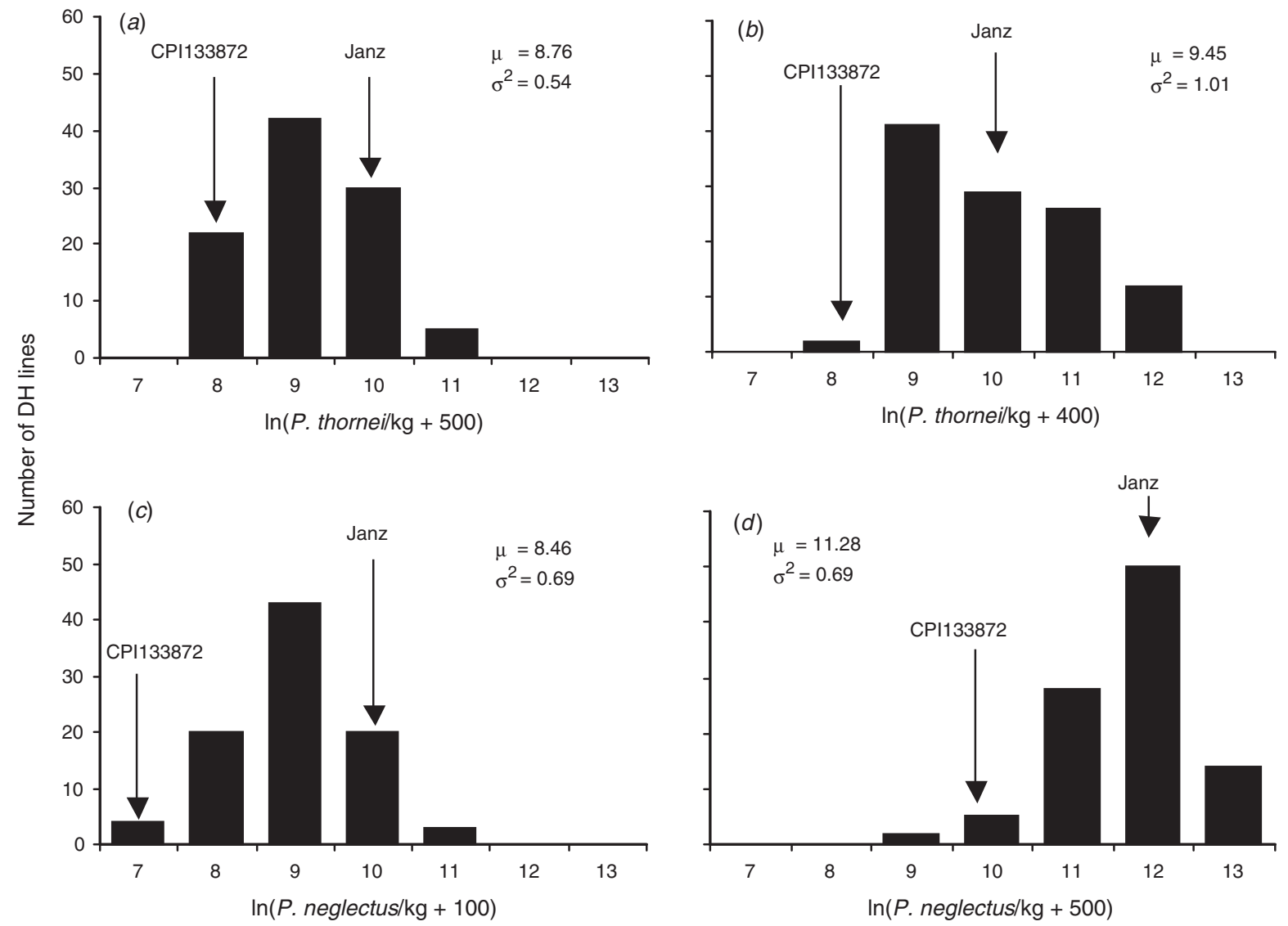

Nematode count

Fig. 1. Resistance to P. thornei and P. neglectus is continuously distributed in the CPI $133872 \times$ Janz doubled-haploid population. (a) Frequency distribution of doubled-haploid lines for resistance to P. thornei in the 2001 glasshouse experiment. (b) Frequency distribution of doubled-haploid lines for resistance to $P$. thornei in the 2002 glasshouse experiment. (c) Frequency distribution of doubled-haploid lines for resistance to P. neglectus in the 2001 glasshouse experiment. (d) Frequency distribution of doubled-haploid lines for resistance to $P$. neglectus in the 2002 glasshouse experiment. 
There was no significant difference between the population mean and the parental means in each of the 4 experiments, suggesting that additive effects are the predominant mode of inheritance for $P$. thornei and $P$. neglectus resistance. There were significant correlations between nematode counts in the 2 years of phenotyping for both $P$. thornei $(r=0.69$, d.f. $=98, P<0.001)$ and $P$. neglectus $(r=0.66$, d.f. $=88, P<0.001)$. There was no correlation between lines resistant to $P$. thornei and lines resistant to P. neglectus in both years (2001: $r=0.23$, d.f. $=89 ; 2002$ : $r=0.22$, d.f. $=89$ ).

\section{Microsatellite primer screen}

Eleven microsatellite markers appeared to be polymorphic between the resistant and susceptible $P$. thornei bulks. These markers were located on chromosomes 2DS, 2DL, 3AS, 3BL, 6DS, 6DL, 7AS, and 7BL. Eight microsatellite markers appeared to be polymorphic between the resistant and susceptible $P$. neglectus bulks. These markers were located on chromosomes 2AS, 2AL, 2BS, 2DS, 5AS, and $5 \mathrm{BL}$. In total, $73 \%$ of the microsatellite primer pairs screened were polymorphic between the synthetic hexaploid parent, CPI133872, and the bread wheat parent, Janz. The polymorphism levels obtained with A-, B-, and Dgenome-specific microsatellite markers were 72,69 , and $81 \%$, respectively.

\section{AFLP primer screen}

The 5 AFLP primer combinations generated 5 bands that were polymorphic between the resistant and susceptible $P$. thornei bulks, and another 6 bands that were polymorphic between the resistant and susceptible P. neglectus bulks. An additional 10 AFLP bands generated by these same 5 primer combinations that were polymorphic between the parents but not the bulks were also scored.

\section{Framework map}

Five marker loci out of 183 polymorphic markers that were scored over the full mapping population had considerable distortion from the expected 1:1 segregation ratio and were subsequently omitted from the dataset. A framework map for CPI133872 $\times$ Janz was constructed using a total of 148 microsatellite and 21 AFLP marker loci. An additional 9 marker loci remained unlinked. The number of markers mapped on the 21 wheat chromosomes ranged from 2 (4D) to 15 (2D) markers. The map covered a total of $2570 \mathrm{cM}$ of the wheat genome. One hundred and sixty-two markers were informative for QTL analysis at an average density of 1 marker per $15.9 \mathrm{cM}$. In general, the microsatellite markers mapped to positions in agreement with published wheat maps.

\section{QTL analysis}

Results of single-marker analysis are shown in Table 1. Seven chromosome regions (2BS, 3AS, 6AS, 6AL, 6DS, 6DL, and 7AS) were detected with putative markers associated with resistance to $P$. thornei. Four chromosome regions (2BS, 4DS, 5AS, and 6DS) were detected with putative markers associated with resistance to $P$. neglectus. Simple interval mapping and composite interval mapping gave similar results and the output of composite interval mapping is shown in Table 2. For all marker-trait associations detected by singlemarker analysis, composite interval mapping identified QTL peaks above LRS of 11.5 (equivalent to LOD score of 2.5) in at least 1 year of phenotypic data.

A major QTL was identified on chromosome 6DS at the distal marker Xbarc183 that was associated with resistance to both $P$. thornei and $P$. neglectus (designated for the 2 traits as QRlnt.lrc-6D.1 and QRlnn.lrc-6D.1). The marker allele inherited from CPI133872 explained 22.0-24.2\% of the phenotypic variation for resistance to $P$. thornei and the allele inherited from Janz explained 11.3-14.0\% of the phenotypic variation for resistance to P. neglectus. A second QTL for resistance to $P$. thornei on chromosome 6DL (QRlnt.lrc-6D.2) was significant in the 2001 data, explaining $13.4-8.3 \%$ of the phenotypic variation. Two other putative QTLs for resistance to $P$. thornei were identified, $Q R \operatorname{lnt}$.lrc2B.1 and QRlnt.lrc-6A.1; however, these QTLs fell below the significance threshold set by the permutation test. All QTLs for resistance to $P$. thornei were inherited from the synthetic hexaploid parent, except for QRInt.lrc-6A.1, which was inherited from Janz.

A second QTL for resistance to P. neglectus, QRlnn.lrc$4 D .1$, was significant only in the 2002 data and explained $10.3-15.4 \%$ of the phenotypic variation. Marker alleles for resistance in this QTL region were inherited from Janz. A significant QTL region on 4BS (QRlnn.lrc$4 B .1$ ) inherited from CPI133872 explained $10.7 \%$ of the phenotypic variation for resistance to $P$. neglectus in 2002 , but was not detected in 2001. Two other minor QTLs for resistance to P. neglectus (QRlnn.lrc-2B.1 and QRlnn.lrc-3D.1) inherited from CPI133872 were detected. Two markers, Xgwm 344 and $X s c h f c 3$, linked to Rlnn 1 in Excalibur- and Krichauff-derived populations were assessed in the CPI133872 $\times$ Janz population. Microsatellite marker Xgwm344 was not polymorphic and Xschfc3 showed no association with resistance to $P$. neglectus in the CPI133872 $\times$ Janz population.

\section{Discussion}

Two major QTLs for resistance to P. thornei, QRlnt.lrc-6D.1 and QRlnt.lrc-6D.2, were detected. The presence of these stable QTLs in 2 years of phenotypic data provides strong evidence that these chromosome regions contain true genetic factors influencing the phenotype. Additional minor QTLs 
Table 1. Single-marker analysis identified putative markers with significant associations with resistance to $P$. thornei and $P$. neglectus in both years of phenotyping

\begin{tabular}{|c|c|c|c|c|c|c|}
\hline \multirow[t]{2}{*}{$\mathrm{Chr}^{\mathrm{A}}$} & \multirow[t]{2}{*}{ Marker } & \multirow{2}{*}{$\begin{array}{l}\text { Position }^{\mathrm{B}} \\
\text { (cM) }\end{array}$} & \multicolumn{2}{|c|}{ LRS for $P$. thornei resistance } & \multicolumn{2}{|c|}{ LRS for $P$. neglectus resistance } \\
\hline & & & 2001 & 2002 & 2001 & 2002 \\
\hline 2BS & $X w m c 025 b$ & 0.0 & $6.8 * *$ & $7.6^{* *}$ & $6.2 *$ & $6.5^{*}$ \\
\hline $3 \mathrm{AS}$ & Xgwm005 & 0.0 & $4.6^{*}$ & $9.9 * *$ & & \\
\hline $3 \mathrm{AS}$ & $X w m c 050$ & 1.2 & $4.3^{*}$ & $7.3 * *$ & & \\
\hline $3 \mathrm{AS}$ & $X g d m 141$ & 4.9 & $5.2 *$ & $13.5 * * *$ & & \\
\hline 4DS & Xwmc331 & 62.7 & & & $6.5^{*}$ & $13.9 * * *$ \\
\hline $5 \mathrm{AS}$ & Xgwm 304 & 30.4 & & & $4.7^{*}$ & $7.1^{* *}$ \\
\hline $6 \mathrm{AS}$ & Xpsp3029 & 0.0 & $12.0 * * *$ & $12.0 * * *$ & & \\
\hline $6 \mathrm{AL}$ & $X w m c 256$ & 107.5 & $7.1 * *$ & $8.7 * *$ & & \\
\hline $6 \mathrm{AL}$ & Xgwm570 & 115.6 & $6.8 * *$ & $7.8 * *$ & & \\
\hline $6 \mathrm{AL}$ & Xwmc201 & 113.3 & $4.5^{*}$ & $5.6^{*}$ & & \\
\hline $6 \mathrm{AL}$ & Xwmc179 & 115.6 & $8.2 * *$ & $9.4^{* *}$ & & \\
\hline $6 \mathrm{AL}$ & P37M54a & 121.0 & $4.5^{*}$ & $8.1^{* *}$ & & \\
\hline 6DS & Xbarc183 & 0.0 & $16.5 * * * *$ & $25.7 * * * *$ & $10.5^{* *}$ & $11.8 * *$ \\
\hline 6DS & $X g d m 132 a$ & 24.4 & $7.9 * *$ & $16.5 * * * *$ & & \\
\hline 6DS & Xgwm469 & 29.7 & $7.7 * *$ & $9.7 * *$ & & \\
\hline 6DS & $\operatorname{Xgdm036}$ & 38.2 & $5.8^{*}$ & $7.5^{* *}$ & & \\
\hline $6 \mathrm{DL}$ & Xgdm098 & 92.0 & $19.2 * * * *$ & $11.3 * * *$ & & \\
\hline $6 \mathrm{DL}$ & Xgpw95010 & 100.8 & $24.5 * * * *$ & $16.0 * * * *$ & & \\
\hline $6 \mathrm{DL}$ & Xbarc021 & 106.3 & $21.3 * * * *$ & $12.8 * * * *$ & & \\
\hline 7AS & $X w m c 283$ & 168.4 & $14.2 * * *$ & $12.2 * * *$ & & \\
\hline
\end{tabular}

Significance levels of likelihood ratio statistic (LRS) values: $* P<0.05 ; * * P<0.01 ; * * * P<0.001 ; * * * P<0.0001$.

${ }^{\mathrm{A}}$ Chromosome.

${ }^{\mathrm{B}}$ Position of marker on chromosome in centiMorgans (cM).

Table 2. Composite interval mapping detected major QTLs for resistance to P. thornei on chromosome arms 6DS and 6DL, and to $P$. neglectus on 6DS and 4DS

\begin{tabular}{|c|c|c|c|c|c|c|c|c|}
\hline \multirow[t]{2}{*}{$\overline{\text { QTL }}$} & \multirow{2}{*}{$\begin{array}{l}\text { Position }^{\mathrm{A}} \\
(\mathrm{cM})\end{array}$} & \multirow{2}{*}{$\begin{array}{l}\text { Flanking } \\
\text { markers }\end{array}$} & \multirow{2}{*}{$\begin{array}{c}\text { Interval } \\
\text { length }^{B} \\
(\mathrm{cM})\end{array}$} & \multicolumn{2}{|c|}{$2001^{\mathrm{C}}$} & \multicolumn{2}{|c|}{$2002^{\mathrm{C}}$} & \multirow{2}{*}{$\begin{array}{l}\text { Allele for } \\
\text { resistance }\end{array}$} \\
\hline & & & & LRS & $R^{2}(\%)$ & LRS & $R^{2}(\%)$ & \\
\hline $\begin{array}{l}\text { QRlnt.lrc-2B.1 } \\
\text { QRlnn.lrc-2B.1 }\end{array}$ & 0.0 & $\begin{array}{l}\text { Xwmc025b } \\
X w m c 154\end{array}$ & 15.8 & $\begin{array}{l}- \\
9.4\end{array}$ & $\begin{array}{c}- \\
11.2\end{array}$ & $\begin{array}{l}15.2 \\
12.4\end{array}$ & $\begin{array}{l}7.4 \\
6.6\end{array}$ & $\begin{array}{l}\text { CPI133872 } \\
\text { CPI133872 }\end{array}$ \\
\hline QRlnn.lrc-3D.1 & 40.2 & $\begin{array}{l}\text { Xgwm161 } \\
\text { Xgwm183 } \\
\text { Xgwm664 }\end{array}$ & $\begin{array}{l}18.9 \\
33.4\end{array}$ & - & - & 12.6 & 11.4 & CPI133872 \\
\hline QRlnn.lrc-4B.1 & 17.4 & $\begin{array}{l}\text { Xgwm066a } \\
\text { Xwmc047 }\end{array}$ & 6.4 & - & - & $17.2 *$ & 10.7 & CPI133872 \\
\hline QRInn.Irc-4D.1 & 50.7 & $\begin{array}{l}X w m c 052 \\
X w m c 331 \\
X b \operatorname{arc} 098\end{array}$ & $\begin{array}{r}10.1 \\
2.4\end{array}$ & 11.6 & 15.4 & $15.8^{*}$ & 10.3 & Janz \\
\hline QRlnt.lrc-6A.1 & 0.0 & $\begin{array}{l}\text { Xpsp3029 } \\
\text { Xgwm459 }\end{array}$ & 50.5 & 11.7 & 7.5 & 11.3 & 9.4 & Janz \\
\hline $\begin{array}{l}\text { QRlnt.lrc-6D.1 } \\
\text { QRlnn.lrc-6D.1 }\end{array}$ & 0.0 & $\begin{array}{l}X b \operatorname{arc} 183 \\
X b \operatorname{arc} 173\end{array}$ & 19.2 & $\begin{array}{l}23.9 * \\
17.3 *\end{array}$ & $\begin{array}{l}22.0 \\
14.0\end{array}$ & $\begin{array}{l}42.5^{*} \\
17.8^{*}\end{array}$ & $\begin{array}{l}24.2 \\
11.3\end{array}$ & $\begin{array}{c}\text { CPI133872 } \\
\text { Janz }\end{array}$ \\
\hline QRlnt.lrc-6D.2 & 66.5 & $\begin{array}{l}\text { Xgdm098 } \\
\text { Xgpw95010 } \\
\text { Xbarc021 }\end{array}$ & $\begin{array}{l}8.8 \\
5.6\end{array}$ & $24.6^{*}$ & 13.4 & 16.5 & 8.3 & CPI133872 \\
\hline
\end{tabular}

$* P<0.05$ for QTLs with LRS value, calculated from permutation tests. The threshold LRS values for resistance to $P$. thornei were 15.8 and 22.3 for 2001 and 2002, respectively. The threshold LRS values for resistance to P. neglectus were 13.5 and 13.1 for 2001 and 2002, respectively.

${ }^{\text {A }}$ Position of marker on chromosome in centiMorgans (cM).

${ }^{B}$ Interval between flanking markers in centiMorgans (cM).

${ }^{\mathrm{C}}$ Peak value of the maximum likelihood ratio statistic (LRS) observed for the QTL region and the proportion of phenotypic variation explained by the QTLs $\left(R^{2}\right)$ are shown for markers with a LRS value of 9.2 (LOD 2.0). 
detected for resistance to $P$. thornei were QRInt.lrc-6A.1 and QRlnt.lrc-2B.1. Chromosomes 6D and 2B have been previously reported to carry QTLs for $P$. thornei resistance (Thompson et al. 1999).

Two major QTLs detected for resistance to P. neglectus were QRlnn.lrc-6D.1 and QRlnn.lrc-4D.1. Additional putative QTLs detected for resistance to $P$. neglectus were QRlnn.lrc-2B.1, QRlnn.lrc-3D.1, and QRlnn.lrc-4B.1. These QTLs identified in the CPI133872 $\times$ Janz population are all novel regions associated with resistance to P. neglectus. In comparison, the only previously mapped $P$. neglectus resistance gene $(R \operatorname{lnn} 1)$ was reported as a monogenic trait located on chromosome 7AL in the wheat cultivar Excalibur (Williams et al. 2002). However, no marker-trait associations were detected with markers on 7AL in the CPI133872 $\times$ Janz population.

Alternative marker alleles of the codominant microsatellite Xbarc183 (located on chromosome 6DS) were associated with resistance to both $P$. thornei and $P$. neglectus. This finding indicates that, for these polygenic traits, it is essential to select the appropriate major resistance allele combinations to successfully breed for dual resistance to both species of root-lesion nematode. This means that to obtain dual resistance, lines that possess QRlnt.lrc-6D.1, inherited from CPI133872, must also possess QRlnn.lrc$4 D .1$, inherited from Janz. Similarly, lines that possess QRlnn.lrc-6D.1, inherited from Janz, require QRlnt.lrc$6 D .2$, inherited from CPI133872. Further work to saturate the map in the 6DS chromosome region is required to determine whether recombination between $Q R \operatorname{lnt} . l r c-6 D .1$ and $Q R \operatorname{lnn} . l r c-6 D .1$ is possible.

The distal marker on chromosome 2BS, Xwmc025b, also had a putative association with resistance to both P. thornei and P. neglectus (QRlnt.lrc-2B.1 and QRlnn.lrc$2 B .1)$. In both cases, the positive association was with the marker allele inherited from CPI133872. Studies have shown that resistance genes are frequently grouped in clusters (Williamson and Hussey 1996) and these clusters tend to be located at the distal ends of the chromosome arms (Li et al. 1999). It is possible that one resistance gene in the 2BS region may be effective against both species of nematode (Rossi et al. 1998), or there may be 2 closely linked resistance genes, with each one conferring a single specific resistance (Takken et al. 1999). However, further mapping to extend the 2BS chromosome arm and to resolve the $15.5-\mathrm{cM}$ distance between markers $X w m c 025 b$ and $X w m c 154$, which flank this QTL region, will be required.

BSA has been used to identify markers linked to important disease resistance traits in wheat (Eastwood et al. 1994; Chagué et al. 1999; Huang et al. 2000). However, even though BSA in the CPI133872 $\times$ Janz population successfully detected a small number of putative markers associated with nematode resistance, the polygenic nature of the resistance traits resulted in a number of single-marker associations that were false positives. As an alternative approach to identifying QTLs, constructing a framework map that represented the genome with a limited number of markers successfully identified major QTL regions for root-lesion nematode resistance.

The ITMI population is the most extensively mapped wheat population to date, with its map containing more than 1000 markers covering $5256 \mathrm{cM}$ (Langridge et al. 2001). Considering that the framework map of CPI133872 $\times$ Janz covers only about half of the wheat genome as described in the ITMI population, the major QTLs detected represent a minimum number of the resistance genes acting against root-lesion nematodes. For this reason, QTLs detected below the threshold LRS value [but above the suggestive LRS value of $9.2(\mathrm{LOD}=2.0)]$, have been presented to indicate the chromosome regions that require further investigation to identify markers more closely linked to the QTLs.

QTLs for resistance to $P$. thornei and $P$. neglectus were inherited from both the synthetic hexaploid parent (CPI133872) and the bread wheat parent (Janz). Although Janz is susceptible to both species of root-lesion nematode, wheat lines considerably more susceptible to root-lesion nematode than Janz do exist (Zwart et al. 2004b). Therefore, it is not surprising that major QTLs for resistance to $P$. neglectus were inherited from Janz. More intriguing is the lack of detection of stable major QTLs for resistance to P. neglectus inherited from the resistant parent CPI133872. This may be attributed to $(i)$ the small population size and incomplete coverage of the CPI133872 $\times$ Janz genetic map, which may have been inadequate to detect some QTLs with strong effects; (ii) resistance to P. neglectus in CPI133872 may be controlled by many QTLs with minor effect, which remain undetected in this study; (iii) environmental influences, which may be more important in the inheritance of $P$. neglectus resistance than $P$. thornei resistance; (iv) epistatic interactions between additive QTLs. Although not all QTLs that influence root-lesion nematode resistance may have been detected in this study, the main QTLs detected are of great interest to wheat breeders.

This study has identified and tagged novel QTLs associated with resistance to both $P$. thornei and P. neglectus that will aid in breeding wheat for dual nematode resistance. The QTLs identified in this study have additive inheritance and thus allow the possibility of marker-assisted selection for nematode resistance to be effective in both early and late generations.

\section{Acknowledgments}

The authors thank N. Howes for help with doubled haploid production, C. Donkin for technical assistance, and D. Butler for statistical assistance. This research was funded by the Grains Research and Development 
Corporation as part of the Australian Winter Cereals Molecular Marker Program. R. Zwart was supported by a Queensland Department of Primary Industries and Fisheries post-graduate scholarship.

\section{References}

Bassam BJ, Caetano-Annollés G (1993) Silver staining of DNA in polyacrylamide gels. Applied Biochemistry and Biotechnology 42, 181-188.

Berry S (2002) INRA-Genoplante SSRs from Simon Berry. http://grain.jouy.inra.fr/ggpages/SSRclub/Berry/Berry.html

Breiman A, Graur D (1995) Wheat evolution. Israel Journal of Plant Sciences 43, 85-98.

Brennan JP, Murray GM (1998) 'Economic importance of wheat diseases in Australia.' (NSW Agriculture: Wagga Wagga, NSW)

Bryan GJ, Collins AJ, Stephenson P, Orry A, Smith JB, Gale MD (1997) Isolation and characterisation of microsatellites from hexaploid bread wheat. Theoretical and Applied Genetics 94, 557-563. doi: $10.1007 / \mathrm{s} 001220050451$

Campbell AW, Daggard G, Békés F, Pedler A, Sutherland MW, Appels R (2001) Targetting AFLP-DNA markers to specific traits and chromosome regions. Australian Journal of Agricultural Research 52, 1153-1161. doi: 10.1071/AR01028

Chagué V, Fahima T, Dahan A, Sun GL, Korol AB, Ronin YI, Grama A, Röder MS, Nevo E (1999) Isolation of microsatellite and RAPD markers flanking the $\operatorname{Yr} 15$ gene of wheat using NILs and bulked segregant analysis. Genome 42, 1050-1056. doi: $10.1139 /$ gen-42-6-1050

Churchill GA, Doerge RW (1994) Empirical threshold values for quantitative trait mapping. Genetics 138, 963-971.

Cregan P (2001) Preliminary BARC SSR maps and primer pair details. http://www.scab.org/pdfs/BARC_SSRs_011101.html

Eastwood RF, Lagudah ES, Appels R (1994) A directed search for DNA sequences tightly linked to cereal cyst nematode resistance genes in Triticum tauschii. Genome 37, 311-319.

Farsi M, Vanstone VA, Fisher JM, Rathjen AJ (1995) Genetic variation in resistance to Pratylenchus neglectus in wheat and triticales. Australian Journal of Experimental Agriculture 35, 597-602.

Gupta PK, Balyan HS, Edwards KJ, Isaac P, Korzun V, et al. (2002) Genetic mapping of 66 new microsatellite (SSR) loci in bread wheat. Theoretical and Applied Genetics 105, 413-422. doi: 10.1007/s00122-002-0865-9

Guyomarc'h H, Sourdille P, Charmet G, Edwards KJ, Bernard M (2002) Characterisation of polymorphic microsatellites from Aegilops tauschii and transferability to the D-genome of bread wheat Theoretical and Applied Genetics 104, 1164-1172.

Huang XQ, Hasam SLK, Zeller FJ, Wenzel G, Mohler V (2000) Molecular mapping of the wheat powdery mildew resistance gene $P m 24$ and marker validation for molecular breeding. Theoretical and Applied Genetics 101, 407-414. doi: $10.1007 / \mathrm{s} 001220051497$

Kammholz SJ, Sutherland MW, Banks PM (1996) Improving the efficiency of haploid wheat production mediated by wide crossing. SABRAO Journal 28, 37-46.

Kosambi DD (1944) The estimation of map distances from recombination values. Annals of Eugenics 12, $172-175$.
Lander ES, Green P, Abrahamson J, Barlow A, Daly MJ, Lincoln SE, Newburg L (1987) MAPMAKER: an interactive computer package for constructing primary genetic linkage maps of experimental and natural populations. Genomics 1, 174-181. doi: 10.1016/08887543(87)90010-3

Langridge P, Lagudah ES, Holton TA, Appels R, Sharp P, Chalmers KJ (2001) Trends in genetic and genome analyses in wheat: a review. Australian Journal of Agricultural Research 52, 1043-1077. doi: 10.1071/AR01082

Li WL, Faris JD, Chittoor JM, Leach JE, Hulbert SH, Liu DJ, Chen PD, Gill BS (1999) Genomic mapping of defense response genes in wheat. Theoretical and Applied Genetics 98, 226-233. doi: $10.1007 / \mathrm{s} 001220051062$

Manly KF, Elliott RW (1991) RI MANAGER, a microcomputer program for analysis of data from recombinant inbred strains. Genome 1, 123-126.

Michelmore RW, Paran I, Kesseli RV (1991) Identification of markers linked to disease-resistance genes by bulked segregant analysis: a rapid method to detect markers in specific genomic regions by using segregating populations. Proceedings of the National Academy of Sciences of the USA 88, 9828-9832.

Pestsova E, Ganal MW, Röder MS (2000) Isolation and mapping of microsatellite markers specific for the Dgenome of bread wheat. Genome 43, 689-697. doi: 10.1139/ gen-43-4-689

Prasad M, Varshney RK, Roy JK, Balyan HS, Gupta PK (2000) The use of microsatellites for detecting DNA polymorphism, genotype identification and genetic diversity in wheat. Theoretical and Applied Genetics 100, 584-592.

Röder MS, Korzun V, Wendehake K, Plaschke J, Tixier MH, Leroy P, Ganal MW (1998) A microsatellite map of wheat. Genetics 149, 2007-2023.

Rogowsky PM, Guidet FLY, Langridge P, Shepherd KW, Koebner RMD (1991) Isolation and characterisation of wheat-rye recombinants involving chromosome arm 1DS of wheat. Theoretical and Applied Genetics 82, 537-544. doi: 10.1007/BF00226788

Rossi M, Goggin FL, Milligan SB, Kaloshian I, Ullman DE, Williamson VM (1998) The nematode resistance gene $M i$ of tomato confers resistance against the potato aphid. Proceedings of the National Academy of Sciences of the USA 95, 9750-9754. doi: 10.1073/pnas.95.17.9750

Stephenson P, Bryan G, Kirby J, Collins A, Devos K, Busso C, Gale M (1998) Fifty new microsatellite loci for the wheat genetic map. Theoretical and Applied Genetics 97, 946-949. doi: 10.1007/s001220050975

Takken FLW, Thomas CM, Joosten MHAJ, Golstein C, Westerink N, Hille J, Nijkamp HJJ, de Wit PJGM, Jones JDG (1999) A second gene at the tomato $C f-4$ locus confers resistance to Cladosporium fulvum through recognition of a novel avirulence determinant. Plant Journal 20, 279-288.

Taylor S, Vanstone V, Ware AH, McKay AC, Szot D, Russ MH (1999) Measuring yield loss in cereals caused by root-lesion nematodes (Pratylenchus neglectus and P. thornei) with and without nematicide. Australian Journal of Agricultural Research 50, 617-622.

Thompson JP, Brennan PS, Clewett TG, Sheedy JG, Seymour NP (1999) Progress in breeding wheat for tolerance and resistance to root-lesion nematode (Pratylenchus thornei). Australasian Plant Pathology 28, $45-52$.

Thompson JP, Clewett TG, O'Reilly MM (1993) Tolerance and resistance in wheat to Pratylenchus thornei. In 'Pratylenchus Workshop, 9th Biennial Conference of the Australasian Plant Pathology Society'. Hobart. pp. 36-41. (Australasian Plant Pathology Society: Hobart, Tas) 
Thompson JP, Haak MI (1997) Resistance to root-lesion nematode (Pratylenchus thornei) in Aegilops tauschii Coss., the D-genome donor to wheat. Australian Journal of Agricultural Research 48, 553-559. doi: 10.1071/A96167

Vos P, Hogers R, Bleeker M, Reijans M, van de Lee T, et al. (1995) AFLP: a new technique for DNA fingerprinting. Nucleic Acids Research 23, 4407-4414.

Wang S, Basten CJ, Zeng Z-B (2004) 'Windows QTL Cartographer 2.0.' (Department of Statistics, North Carolina State University: Raleigh, NC)

Williams KJ, Taylor SP, Bogacki P, Pallotta M, Bariana HS, Wallwork H (2002) Mapping of the root-lesion nematode (Pratylenchus neglectus) resistance gene Rlnn1 in wheat. Theoretical and Applied Genetics 104, 874-879. doi: 10.1007/s00122-001-0839-3
Williamson VM, Hussey RS (1996) Nematode pathogenesis and resistance in plants. The Plant Cell 8, 1735-1745. doi: $10.1105 /$ tpc. 8.10 .1735

Zwart RS, Thompson JP, Godwin ID (2004a) Genetic analysis of resistance to root-lesion nematode (Pratylenchus thornei Sher and Allen) in wheat. Plant Breeding 123, 209-212. doi: 10.1111/j.14390523.2004.00986.x

Zwart RS, Thompson JP, Williamson PM, Seymour NP (2004b) Elite sources of resistance in wheat to root-lesion nematode (Pratylenchus thornei and Pratylenchus neglectus) and yellow spot (Pyrenophora tritici-repentis. In 'Proceedings of the 3rd Australasian Soilborne Diseases Symposium'. pp. 220. (South Australian Research and Development Institute: Adelaide, S. Aust.)

Manuscript received 15 September 2004, accepted 10 February 2005 